J. Nonlinear Var. Anal. 3 (2019), No. 2, pp. 189-203

Available online at http://jnva.biemdas.com

https://doi.org/10.23952/jnva.3.2019.2.08

\title{
CONVERGENCE THEOREMS OF COMMON SOLUTIONS FOR FIXED POINT, VARIATIONAL INEQUALITY AND EQUILIBRIUM PROBLEMS
}

\author{
NASEER SHAHZAD ${ }^{1, *}$, HABTU ZEGEYE $^{2}$ \\ ${ }^{1}$ Department of Mathematics, King Abdulaziz University, Jeddah 21589, Saudi Arabia \\ ${ }^{2}$ Department of Mathematics, Botswana International University of Science and Technology, Palapye, Botswana \\ Dedicated to Professor Wataru Takahashi on the occasion of his 75th birthday
}

\begin{abstract}
The aim of this paper is to introduce an iterative process for a common solution of fixed point problem of a continuous pseudocontractive mapping, a variational inequality problem and an equilibrium problem provided their common solution exists. Moreover, a numerical example which supports our main convergence results is presented.
\end{abstract}

Keywords. Equilibrium problem; Fixed point; Monotone mapping; Strong convergence; Variational inequality problem.

2010 Mathematics Subject Classification. 47H05, 47H09, 47J25.

\section{INTRODUCTION}

Let $C$ be a nonempty subset of a real Hilbert space $H$. A mapping $A: C \rightarrow H$ is said to be $\gamma$-inverse strongly monotone if there exists a positive real number $\gamma$ such that

$$
\langle x-y, A x-A y\rangle \geq \gamma\|A x-A y\|^{2}, \quad \forall x, y \in C .
$$

If $A$ is $\gamma$-inverse strongly monotone, then it is Lipschitz continuous with constant $\frac{1}{\gamma}$, i.e.,

$$
\|A x-A y\| \leq \frac{1}{\gamma}\|x-y\|, \quad \forall x, y \in C .
$$

$A$ is said to be strongly monotone if there exists $k>0$ such that

$$
\langle x-y, A x-A y\rangle \geq k\|x-y\|^{2}, \quad \forall x, y \in C .
$$

$A$ is said to be monotone if

$$
\langle x-y, A x-A y\rangle \geq 0, \quad \forall x, y \in C .
$$

Apart from being an important generalization of strongly monotone and $\gamma$-inverse strongly monotone mappings, interest in monotone mappings stems mainly from their firm connection with the important class of nonlinear pseudocontractive mappings. Recall that a mapping $T: C \rightarrow H$ is said to be pseudocontractive if

$$
\langle T x-T y, x-y\rangle \leq\|x-y\|^{2}, \quad \forall x, y \in C .
$$

${ }^{*}$ Corresponding author.

E-mail addresses: nshahzad@kau.edu.sa (N. Shahzad), habtuzh@yahoo.com (H. Zegeye).

Received March 30, 2019; Accepted 15 May, 2019.

(C)2019 Journal of Nonlinear and Variational Analysis 
$T$ is said to be strongly pseudocontractive if there exists $k \in(0,1)$ such that

$$
\langle x-y, T x-T y\rangle \leq k\|x-y\|^{2}, \quad \forall x, y \in C .
$$

$T$ is said to be $k$-strict pseudocontractive if there exists a constant $0 \leq k<1$ such that

$$
\langle x-y, T x-T y\rangle \leq\|x-y\|^{2}-k\|(I-T) x-(I-T) y\|^{2}, \quad \forall x, y \in C .
$$

A mapping $T: C \rightarrow H$ is said to be $L$-Lipschitz if there exits $L \geq 0$ such that

$$
|| T x-T y|\leq L| \mid x-y \|, \quad \forall x, y \in C .
$$

If $L=1$ then $A$ is said to be nonexpansive and if $L<1$, then $A$ is said to be contractive.

We observe that the class of pseudocontractive mappings includes the class of nonexpansive mappings, and $T$ is pseudocontractive if and only if $A:=I-T$ is monotone. Thus the fixed point set of $T, F(T):=$ $\{x \in D(T): T x=x\}$, is the zero point sete of $A, N(A):=\{x \in D(A): A x=0\}$.

Suppose that $A$ is a monotone mapping from $C$ to $H$. The variational inequality problem is formulated as finding

$$
\text { a point } u \in C \text { such that }\langle v-u, A u\rangle \geq 0 \text {, for all } v \in C \text {. }
$$

The set of solutions of the variational inequality problem is denoted by $\operatorname{VI}(C, A)$.

Variational inequality problem is connected with the convex minimization problem, the complementarity problem, the problem of finding a point $u \in C$ satisfying $0 \in A u$. It was initially studied in [7,9] and ever since considerable research efforts have been devoted to iterative methods for approximating solutions of variational inequality and/or fixed points of $T$, when $T$ is nonexpansive or its generalizations; see, for example, $[5,6,20,21]$ and the references contained therein.

In [3], Iiduka, Takahashi and Toyoda studied the projection algorithm given by

$$
x_{n+1}=P_{C}\left(x_{n}-\alpha_{n} A x_{n}\right), \quad \forall n \geq 1,
$$

with an initial point $x_{1} \in C$, where $P_{C}$ is the metric projection from $H$ onto $C$ and $\left\{\alpha_{n}\right\}$ is a sequence of positive real numbers. They proved that if $A$ is $\gamma$-inverse strongly monotone, then the sequence $\left\{x_{n}\right\}$ generated by (1.2) converges weakly to some element of $\operatorname{VI}(C, A)$. They also studied the following iterative scheme:

$$
\left\{\begin{array}{l}
x_{0} \in C, \text { chosen arbitrarily, } \\
y_{n}=P_{C}\left(x_{n}-\alpha_{n} A x_{n}\right), \\
C_{n}=\left\{z \in C:\left\|y_{n}-z\right\| \leq\left\|x_{n}-z\right\|\right\} \\
Q_{n}=\left\{z \in C:\left\langle x_{n}-z, x_{0}-x_{n}\right\rangle \geq 0\right\} \\
x_{n+1}=P_{C_{n} \cap Q_{n}}\left(x_{0}\right), \quad n \geq 1,
\end{array}\right.
$$

where $\left\{\alpha_{n}\right\}$ is a sequence in $[0,2 \gamma]$. They proved that the sequence $\left\{x_{n}\right\}$ generated by (1.3) converges strongly to $P_{V I(C, A)}\left(x_{0}\right)$, where $P_{V I(C, A)}$ is the metric projection from $H$ onto $V I(C, A)$ provided that $A$ is $\gamma$-inverse strongly monotone.

Recently, the problem of finding a common element in the fixed point set of a nonexpansive mapping and the solution set of a variational inequality problem for a $\gamma$-inverse strongly monotone mapping has been considered by many authors; see, for example, $[2,7,8,10,18,19]$ and the references therein. 
In 2005, Iiduka and Takahashi [4] considered a common element problem for a fixed point problem of nonexpansive mappings and a variational inequality problem via the following iterative algorithm:

$$
x_{0}=x \in C, x_{n+1}=\alpha_{n} x+\left(1-\alpha_{n}\right) T P_{C}\left(x_{n}-\lambda_{n} A x_{n}\right), \quad n \geq 0,
$$

where $T: C \rightarrow C$ is a nonexpansive mapping, $A: C \rightarrow H$ is a $\gamma$-inverse strongly monotone mapping, $\left\{\gamma_{n}\right\}$ is a sequence in $(0,1)$, and $\left\{\lambda_{n}\right\}$ is a sequence in $(0,2 \gamma)$. They proved that the sequence $\left\{x_{n}\right\}$ strongly converges to some point $z \in F(T) \cap V I(C, A)$.

Recently, Zegeye and Shahzad [25] investigated the problem of finding a common element in fixed point sets of a Lipschitz pseudocontractive mapping $T$ and solution sets of a variational inequality problem of a $\gamma$-inverse strongly monotone mapping $A$ by considering the following iterative algorithm:

$$
\left\{\begin{array}{l}
y_{n}=\left(1-\beta_{n}\right) x_{n}+\beta_{n} T x_{n}, \\
x_{n+1}=P_{C}\left[\left(1-\alpha_{n}\right)\left(\delta_{n} x_{n}+\theta_{n} T y_{n}+\gamma_{n} P_{C}[I-\gamma A] x_{n}\right)\right],
\end{array}\right.
$$

where $\left\{\delta_{n}\right\},\left\{\theta_{n}\right\},\left\{\gamma_{n}\right\},\left\{\alpha_{n}\right\},\left\{\beta_{n}\right\}$ are in $(0,1)$ satisfying certain conditions. Then, they proved that the sequence $\left\{x_{n}\right\}$ converges strongly to the minimum-norm point of $F(T) \cap V I(C, A)$.

In this paper, one of our concerns is the following:

Question 1. Is it possible to construct an iterative scheme which converges strongly to a common element in the fixed point set of a pseudocontractive mapping and the solution set of a variational inequality problem of a monotone mapping?

Let $f: C \times C \rightarrow \mathbb{R}$ be a bifunction, where $\mathbb{R}$ is the set of real numbers. The equilibrium problem for $f$ is to

$$
\text { find } x^{*} \in C \text { such that } f\left(x^{*}, y\right) \geq 0, \quad \forall y \in C \text {. }
$$

The set of solutions of (1.4) is denoted by $E P(f)$. A number of real world problems can be investigated via the framework of the equilibrium problem; see, e.g., $[1,10]$.

For studying equilibrium problem (1.4), we assume that $f$ satisfies the following conditions:

(A1) $f(x, x)=0$ for all $x \in C$,

(A2) $\mathrm{f}$ is monotone, i.e, $f(x, y)+f(y, x) \leq 0$ for all $x, y \in C$,

(A3) for each $x, y, z \in C, \lim _{t \rightarrow 0} f(t z+(1-t) x, y) \leq f(x, y)$,

(A4) for each $x \in C, y \rightarrow f(x, y)$ is convex and lower semicontinuous.

Recently, many authors have considered the problem of finding a common element in the fixed point set of a nonexpansive mapping, the solution set of an equilibrium problem and the solution set of a variational inequality problem of $\gamma$-inverse strongly monotone mappings; see, e.g. $[8,13,15,16,20$, 21] and the references therein. In [15], Tada and Takahashi investigated fixed points of nonexpansive mappings and solutions of equilibrium problem (1.4). They obtained the following result.

Let $f$ be a bifunction from $C \times C$ into $\mathbb{R}$ satisfying (A1)-(A4), and let $S$ be a nonexpansive mapping of $C$ into $H$ such that $F(S) \cap E P(F) \neq \emptyset$. Let $\left\{x_{n}\right\}$ and $\left\{u_{n}\right\}$ be sequences given by

$$
\left\{\begin{array}{l}
x_{0} \in C, \text { chosen arbitrarily, } \\
u_{n} \in C, \text { such that } f\left(u_{n}, y\right)+\frac{1}{r_{n}}\left\langle y-u_{n}, u_{n}-x_{n}\right\rangle \geq 0, \forall y \in C, \\
x_{n+1}=\alpha_{n} x_{n}+\left(1-\alpha_{n}\right) S u_{n}, n \geq 0,
\end{array}\right.
$$


where $\left\{\alpha_{n}\right\} \subset[a, b]$ for some $a, b \in(0,1)$ and $\left\{r_{n}\right\} \subset(0, \infty)$ satisfies $\liminf _{n \rightarrow \infty} r_{n}>0$. Then $\left\{x_{n}\right\}$ converges weakly to $w \in F(S) \cap E P(F)$, where $w=\lim _{n \rightarrow \infty} F(S) \cap E P(F) x_{n}$.

In connection with the strong convergence, Tada and Takahashi [15] also introduced the following iterative scheme for approximating the common element. Their algorithm is as follows.

$$
\left\{\begin{array}{l}
x_{0} \in C, \text { chosen arbitrarily, } \\
u_{n} \in C, \text { such that } f\left(u_{n}, y\right)+\frac{1}{r_{n}}\left\langle y-u_{n}, u_{n}-x_{n}\right\rangle \geq 0, \forall y \in C, \\
w_{n}=\left(1-\alpha_{n}\right) x_{n}+\alpha_{n} T u_{n}, \\
C_{n}=\left\{z \in H:\left\|w_{n}-z\right\| \leq\left\|x_{n}-z\right\|\right\}, \\
Q_{n}=\left\{z \in C:\left\langle x_{n}-z, x_{0}-x_{n}\right\rangle \geq 0\right\}, \\
x_{n+1}=P_{C_{n} \cap Q_{n}}\left(x_{0}\right), n \geq 0,
\end{array}\right.
$$

where $\left\{\alpha_{n}\right\} \subset[a, b]$ for some $a, b \in(0,1)$ and $\left\{r_{n}\right\} \subset(0, \infty)$ satisfies $\liminf _{n \rightarrow \infty} r_{n}>0$. They proved that $\left\{x_{n}\right\}$ and $\left\{u_{n}\right\}$ converge strongly to $z \in E P(f) \cap F(T)$, where $z=P_{E P(f) \cap F(T)}\left(x_{0}\right)$.

For finding an element in $F(T) \cap V I(C, A) \cap E P(f)$, Kumam [8] introduced the following iterative scheme:

$$
\left\{\begin{array}{l}
x_{0} \in C, \text { chosen arbitrarily, } \\
u_{n} \in C, \text { such that } f\left(u_{n}, y\right)+\frac{1}{r_{n}}\left\langle y-u_{n}, u_{n}-x_{n}\right\rangle \geq 0, \forall y \in C, \\
w_{n}=\alpha_{n} x_{n}+\left(1-\alpha_{n}\right) T P_{C}\left(u_{n}-\lambda_{n} A u_{n}\right), \\
C_{n}=\left\{z \in H:\left\|w_{n}-z\right\| \leq\left\|x_{n}-z\right\|\right\}, \\
Q_{n}=\left\{z \in C:\left\langle x_{n}-z, x_{0}-x_{n}\right\rangle \geq 0\right\}, \\
x_{n+1}=P_{C_{n} \cap Q_{n}}\left(x_{0}\right), n \geq 0,
\end{array}\right.
$$

where $A: C \rightarrow H$ is a $\gamma$-inverse strongly monotone mapping and $T$ is a nonexpansive mapping. They proved that $\left\{x_{n}\right\}$ and $\left\{u_{n}\right\}$ converge strongly to $z \in F(T) \cap V I(C, A) \cap E P(f)$.

We remark that the computation of $x_{n+1}$ in Algorithms (1.5) and (1.6) are not simple in applications because of the involvement of computation of $C_{n+1}$ from $C_{n}$ for each $n \geq 1$.

This brings us to the second concern in this paper

Question 2. Can we construct an iterative scheme for a common element in the fixed point set of a pseudocontractive mapping, the solution set of a variational inequality problem for a monotone mapping and the solution set of an equilibrium problem?

It is our purpose in this paper to introduce an iterative scheme $\left\{x_{n}\right\}$ which converges strongly to a common element in the fixed point set of a continuous pseudoconcontractive mapping, the solution set of a variational inequality problem for a Lipschitz monotone mapping and the solution set of an equilibrium problem. In addition, a numerical example which supports our main convergence result is presented. Our scheme does not involve computation of $C_{n}$ and $Q_{n}$ to obtain $x_{n+1}$ for each $n \geq 1$. Our theorems extend and unify most of the results that have been proved for this important class of nonlinear mappings. 


\section{PRELIMINARIES}

Let $C$ be a nonempty, closed and convex subset of a real Hilbert space $H$. We recall that for each point $x \in H$, there exists a unique nearest point in $C$, denoted by $P_{C} x$, satisfying

$$
\left\|x-P_{C} x\right\| \leq\|x-y\|, \quad \forall y \in C .
$$

The mapping $P_{C}$ is called the metric projection of $H$ onto $C$. We know that $P_{C}$ is a nonexpansive mapping and is characterized by the following property (see, e.g., [17])

$$
\left\|y-P_{C} x\right\|^{2} \leq\|x-y\|^{2}-\left\|x-P_{C} x\right\|^{2}, \quad \forall x \in H, y \in C .
$$

In the sequel, we shall make use of the following lammas.

Lemma 2.1. [24] Let $C$ be a nonempty closed and convex subset of a real Hilbert space $H$. If $A: C \rightarrow H$ is continuous monotone mapping, then $\operatorname{VI}(C, A)$ is closed and convex.

Lemma 2.2. [23] Let $H$ be a real Hilbert space. Then for all $x, y \in H$ and $\alpha \in[0,1]$ the following equality holds:

$$
\|\alpha x+(1-\alpha) y\|^{2}=\alpha\|x\|^{2}+(1-\alpha)\|y\|^{2}-\alpha(1-\alpha)\|x-y\|^{2} .
$$

Lemma 2.3. [17] Let $H$ be a real Hilbert space. Then for any given $x, y \in H$, the following inequality holds:

$$
\|x+y\|^{2} \leq\|x\|^{2}+2\langle y, x+y\rangle .
$$

Lemma 2.4. [17] Let $C$ be a nonempty, closed and convex subset of a real Hilbert space $H$ and $x \in H$. Then $x_{0}=P_{C} x$ if and only if

$$
\left\langle z-x_{0}, x-x_{0}\right\rangle \leq 0, \quad \forall z \in C
$$

Lemma 2.5. [18] Let $\left\{a_{n}\right\}$ be a sequence of nonnegative real numbers satisfying the following relation:

$$
a_{n+1} \leq\left(1-\alpha_{n}\right) a_{n}+\alpha_{n} \delta_{n}, \quad n \geq n_{0},
$$

where $\left\{\alpha_{n}\right\} \subset(0,1)$ and $\left\{\delta_{n}\right\} \subset \mathbb{R}$ satisfying the following conditions: $\lim _{n \rightarrow \infty} \alpha_{n}=0, \sum_{n=1}^{\infty} \alpha_{n}=\infty$, and $\limsup \sup _{n \rightarrow \infty} \delta_{n} \leq 0$. Then, $\lim _{n \rightarrow \infty} a_{n}=0$.

Lemma 2.6. [11] Let $\left\{a_{n}\right\}$ be sequences of real numbers such that there exists a subsequence $\left\{n_{i}\right\}$ of $\{n\}$ such that $a_{n_{i}}<a_{n_{i}+1}$, for all $i \in \mathbb{N}$. Then there exists a nondecreasing sequence $\left\{m_{k}\right\} \subset \mathbb{N}$ such that $m_{k} \rightarrow \infty$ and the following properties are satisfied by all (sufficiently large) numbers $k \in \mathbb{N}$ :

$$
a_{m_{k}} \leq a_{m_{k}+1} \text { and } a_{k} \leq a_{m_{k}+1} .
$$

In fact, $m_{k}=\max \left\{j \leq k: a_{j}<a_{j+1}\right\}$.

Lemma 2.7. [22] Let $C$ be a nonempty, closed, and convex subset of a real Hilbert space $H$. Let $T: C \rightarrow$ $H$ be a continuous pseudocontractive mapping. For $r>0$ and $x \in E$, define a mapping $T_{r}: E \rightarrow C$ as follows:

$$
T_{r} x:=\left\{z \in C:\langle y-z, T z\rangle-\frac{1}{r}\langle y-z,(1+r) z-x\rangle \leq 0, \forall y \in C\right\} .
$$

Then the following hold:

(C1) $T_{r}$ is single-valued;

(C2) $F\left(T_{r}\right)=F(T)$; 
(C3) $F(T)$ is closed and convex.

(C4) $\left\|T_{r} x-p\right\|^{2}+\left\|T_{r} x-x\right\|^{2} \leq\|x-p\|^{2}, \forall p \in F\left(T_{r}\right), x \in H$.

Lemma 2.8. [16] Let $C$ be a nonempty, closed and convex subset of a real Hilbert space $H$. Let $f$ be a bifunction from $C \times C$ to $\mathbb{R}$ satisfying (Al)-(A4). For $r>0$ and $x \in H$, define a mapping $F_{r}: H \rightarrow C$ as follows:

$$
F_{r} x:=\left\{z \in C: f(z, y)+\frac{1}{r}\langle y-z, z-x\rangle \geq 0, \forall y \in C\right\}
$$

for all $x \in E$. Then the following hold:

(1) $F_{r}$ is single-valued;

(2) $F\left(F_{r}\right)=E P(f)$;

(3) $E P(f)$ is closed and convex;

(4) $\left\|F_{r} x-p\right\|^{2}+\left\|F_{r} x-x\right\|^{2} \leq\|x-p\|^{2}, \forall p \in F\left(F_{r}\right), x \in H$.

\section{MAIN RESULTS}

Let $C$ be a nonempty, closed and convex subset of a real Hilbert space $H$. Let $T: C \rightarrow H$ be a continuous pseudocontractive mapping and let $f: C \times C \rightarrow \mathbb{R}$ be a bifunction satisfying (A1)-(A4). In what follows, $T_{r_{n}}, F_{r_{n}}: H \rightarrow C$ are defined as follows.

For $x \in H$ and $\left\{r_{n}\right\} \subset(0, \infty)$ satisfying $\inf _{n \rightarrow \infty} r_{n}>0$, define

$$
T_{r_{n}} x:=\left\{z \in C:\langle y-z, T z\rangle-\frac{1}{r_{n}}\left\langle y-z,\left(1+r_{n}\right) z-x\right\rangle \leq 0, \forall y \in C\right\}
$$

and

$$
F_{r_{n}} x:=\left\{z \in C: f(z, y)+\frac{1}{r_{n}}\langle y-z, z-x\rangle \geq 0, \forall y \in C\right\} .
$$

For the rest of this paper, $P_{C}$ is the metric projection from $H$ onto $C$ and $\left\{\alpha_{n}\right\} \subset(0, c] \subset(0,1)$ for all $n \geq 0$ satisfying $\lim _{n \rightarrow \infty} \alpha_{n}=0$ and $\sum_{n=0}^{\infty} \alpha_{n}=\infty$.

Now, we prove our main convergence theorem.

Theorem 3.1. Let $C$ be a nonempty, closed and convex subset of a real Hilbert space H. Let $T: C \rightarrow H$ be a continuous pseudocontractive mapping and let $A: C \rightarrow H$ be a L-Lipschitz monotone mapping with Lipschitz constant L. Let $f: C \times C \rightarrow \mathbb{R}$ be a bifunction satisfying (A1)-(A4). Assume that $\mathscr{F}=$ $F(T) \cap V I(C, A) \cap E P(f)$ is nonempty. Let $\left\{x_{n}\right\}$ be a sequence generated from an arbitrary $x_{0}, u \in C$ by

$$
\left\{\begin{array}{l}
z_{n}=P_{C}\left[x_{n}-\gamma_{n} A x_{n}\right], \\
x_{n+1}=\alpha_{n} u+\left(1-\alpha_{n}\right)\left[\beta_{n} y_{n}+\left(1-\beta_{n}\right) u_{n}\right],
\end{array}\right.
$$

where $y_{n}=F_{r_{n}} T_{r_{n}} x_{n}, u_{n}=P_{C}\left[x_{n}-\gamma_{n} A z_{n}\right],\left\{\gamma_{n}\right\} \subset[a, b] \subset\left(0, \frac{1}{L}\right)$ and $\left\{\beta_{n}\right\} \subset[e, 1) \subset(0,1)$. Then, $\left\{x_{n}\right\}$ converges strongly to a point $x^{*}$ in $\mathscr{F}$ which is nearest to $u$.

Proof. Let $p \in \mathscr{F}$ and $v_{n}=T_{r_{n}} x_{n}$. Then, we get that $y_{n}=F_{r_{n}} v_{n}$. From Lemmas 2.7 and 2.8 we have

$$
\begin{aligned}
\left\|y_{n}-v\right\|^{2} & =\left\|F_{r_{n}} v_{n}-F_{r_{n}} p\right\|^{2} \\
& \leq\left\|v_{n}-p\right\|^{2}-\left\|v_{n}-y_{n}\right\|^{2} \\
& \leq\left\|x_{n}-p\right\|^{2}-\left\|v_{n}-x_{n}\right\|^{2}-\left\|v_{n}-y_{n}\right\|^{2} \leq\left\|x_{n}-p\right\|^{2} .
\end{aligned}
$$


From (2.1) and (3.1) we get

$$
\begin{aligned}
\left\|u_{n}-p\right\|^{2} \leq & \left\|x_{n}-\gamma_{n} A z_{n}-p\right\|^{2}-\left\|x_{n}-\gamma_{n} A z_{n}-u_{n}\right\|^{2} \\
= & \left\|x_{n}-p\right\|^{2}-\left\|x_{n}-u_{n}\right\|^{2}+2 \gamma_{n}\left\langle A z_{n}, p-u_{n}\right\rangle \\
= & \left\|x_{n}-p\right\|^{2}-\left\|x_{n}-u_{n}\right\|^{2}+2 \gamma_{n}\left(\left\langle A z_{n}-A p, p-z_{n}\right\rangle\right. \\
& \left.+\left\langle A p, p-z_{n}\right\rangle+\left\langle A z_{n}, z_{n}-u_{n}\right\rangle\right) \\
\leq & \left\|x_{n}-p\right\|^{2}-\left\|x_{n}-u_{n}\right\|^{2}+2 \gamma_{n}\left\langle A z_{n}, z_{n}-u_{n}\right\rangle \\
= & \left\|x_{n}-p\right\|^{2}-\left\|x_{n}-z_{n}\right\|^{2}-\left\|z_{n}-u_{n}\right\|^{2} \\
& +2\left\langle\gamma_{n} A z_{n}-x_{n}+z_{n}, z_{n}-u_{n}\right\rangle .
\end{aligned}
$$

Using the Lipschitz property of $A$, we obtain

$$
\begin{aligned}
\left\langle\gamma_{n} A z_{n}-x_{n}+z_{n}, z_{n}-u_{n}\right\rangle & =\left\langle x_{n}-\gamma_{n} A x_{n}-z_{n}, u_{n}-z_{n}\right\rangle+\left\langle\gamma_{n} A x_{n}-\gamma_{n} A z_{n}, u_{n}-z_{n}\right\rangle \\
& \leq\left\langle\gamma_{n} A x_{n}-\gamma_{n} A z_{n}, u_{n}-z_{n}\right\rangle \\
& \leq \gamma_{n} L|| x_{n}-z_{n}|||| u_{n}-z_{n} \| .
\end{aligned}
$$

Thus, from (3.3) and (3.4), we obtain

$$
\begin{aligned}
\left\|u_{n}-p\right\|^{2} \leq & \left\|x_{n}-p\right\|^{2}-\left\|x_{n}-z_{n}\right\|^{2}-\left\|z_{n}-u_{n}\right\|^{2} \\
& +2 \gamma_{n} L\left\|x_{n}-z_{n}\left|\left\||| u_{n}-z_{n}\right\|\right.\right. \\
\leq & \left\|x_{n}-p\right\|^{2}-\left\|x_{n}-z_{n}\right\|^{2}-\left\|z_{n}-u_{n}\right\|^{2} \\
& +\gamma_{n} L\left(\left\|x_{n}-z_{n}\right\|^{2}+\left\|z_{n}-u_{n}\right\|^{2}\right) \\
\leq & \left\|x_{n}-p\right\|^{2}+\left(\gamma_{n} L-1\right)\left[\left\|x_{n}-z_{n}\right\|^{2}+\left\|z_{n}-u_{n}\right\|^{2}\right. \\
\leq & \left\|x_{n}-p\right\|^{2} .
\end{aligned}
$$

Using (3.1), (3.2), (3.5), Lemma 2.2 and the fact that $L \gamma_{n}<1$, we have

$$
\begin{aligned}
\left\|x_{n+1}-p\right\|^{2}= & \left\|\alpha_{n} u+\left(1-\alpha_{n}\right)\left[\beta_{n} y_{n}+\left(1-\beta_{n}\right) u_{n}\right]-p\right\|^{2} \\
= & \left\|\alpha_{n}(u-p)+\left(1-\alpha_{n}\right)\left(\left[\beta_{n} y_{n}+\left(1-\beta_{n}\right) u_{n}\right]-p\right)\right\|^{2} \\
\leq & \alpha_{n}\|u-p\|^{2}+\left(1-\alpha_{n}\right) \beta_{n}\left\|y_{n}-p\right\|^{2} \\
& +\left(1-\alpha_{n}\right)\left(1-\beta_{n}\right)\left\|u_{n}-p\right\|^{2} \\
\leq & \alpha_{n}\|u-p\|^{2}+\left(1-\alpha_{n}\right) \beta_{n}\left\|x_{n}-p\right\|^{2} \\
& +\left(1-\alpha_{n}\right)\left(1-\beta_{n}\right)\left\|x_{n}-p\right\|^{2} \\
\leq & \alpha_{n}\|u-p\|^{2}+\left(1-\alpha_{n}\right)\left\|x_{n}-p\right\|^{2} .
\end{aligned}
$$

Therefore, by induction, we get that

$$
\left\|x_{n+1}-p\right\|^{2} \leq \max \left\{\|u-p\|^{2},\left\|x_{0}-p\right\|^{2}\right\}, \quad \forall n \geq 0,
$$

which implies that $\left\{x_{n}\right\},\left\{y_{n}\right\},\left\{z_{n}\right\}$ and $\left\{u_{n}\right\}$ are bounded. 
Let $x^{*}=\Pi_{\mathscr{F}} u$. From (3.1), (3.2), (3.5), Lemma 2.2 and 2.3, and the fact that $L \gamma_{n}<1$, we obtain

$$
\begin{aligned}
\left\|x_{n+1}-x^{*}\right\|^{2}= & \left\|\alpha_{n} u+\left(1-\alpha_{n}\right)\left[\beta_{n} y_{n}+\left(1-\beta_{n}\right) u_{n}\right]-x^{*}\right\|^{2} \\
= & \| \alpha_{n}\left(u-x^{*}\right)+\left.\left(1-\alpha_{n}\right)\left[\beta_{n} y_{n}+\left(1-\beta_{n}\right) u_{n}-x^{*}\right]\right|^{2} \\
\leq & \left(1-\alpha_{n}\right)\left\|\beta_{n}\left(y_{n}-x^{*}\right)+\left(1-\beta_{n}\right)\left(u_{n}-x^{*}\right)\right\|^{2} \\
& +2\left\langle x_{n+1}-x^{*}, \alpha_{n}\left(u-x^{*}\right)\right\rangle \\
\leq & \left(1-\alpha_{n}\right)\left[\beta_{n}\left\|y_{n}-x^{*}\right\|^{2}+\left(1-\beta_{n}\right)\left\|u_{n}-x^{*}\right\|^{2}\right] \\
& +2 \alpha_{n}\left\langle x_{n+1}-x^{*}, u-x^{*}\right\rangle \\
\leq & \left(1-\alpha_{n}\right) \beta_{n}\left(\left\|x_{n}-x^{*}\right\|^{2}-\left\|v_{n}-x_{n}\right\|^{2}-\left\|v_{n}-y_{n}\right\|^{2}\right) \\
& +\left(1-\alpha_{n}\right)\left(1-\beta_{n}\right)\left(\left\|x_{n}-x^{*}\right\|^{2}+\left(L \gamma_{n}-1\right)\left[\left\|x_{n}-z_{n}\right\|^{2}+\left\|z_{n}-u_{n}\right\|^{2}\right]\right) \\
& +2 \alpha_{n}\left\langle x_{n+1}-x^{*}, u-x^{*}\right\rangle .
\end{aligned}
$$

Hence

$$
\begin{aligned}
\left\|x_{n+1}-x^{*}\right\|^{2} \leq & \left(1-\alpha_{n}\right)\left\|x_{n}-x^{*}\right\|^{2}-\left(1-\alpha_{n}\right) \beta_{n}\left(\left\|v_{n}-x_{n}\right\|^{2}+\left\|v_{n}-y_{n}\right\|^{2}\right) \\
& +\left(1-\alpha_{n}\right)\left(1-\beta_{n}\right)\left(L \gamma_{n}-1\right)\left[\left\|x_{n}-z_{n}\right\|^{2}+\left\|z_{n}-u_{n}\right\|^{2}\right] \\
& +2 \alpha_{n}\left\langle x_{n+1}-x^{*}, u-x^{*}\right\rangle \\
\leq & \left(1-\alpha_{n}\right)\left\|x_{n}-x^{*}\right\|^{2}+2 \alpha_{n}\left\|x_{n+1}-x_{n}\right\|\left\|u-x^{*}\right\| \\
& +2 \alpha_{n}\left\langle x_{n}-x^{*}, u-x^{*}\right\rangle .
\end{aligned}
$$

Next, we consider two cases.

Case 1. Suppose that there exists $n_{0} \in \mathbb{N}$ such that $\left\{\left\|x_{n}-x^{*}\right\|\right\}$ is decreasing for all $n \geq n_{0}$. Then, we get that, $\left\{\left\|x_{n}-x^{*}\right\|\right\}$ is convergent. Thus, from (3.7) and the fact that $\gamma_{n}<b<1$ for all $n \geq 0$ and $\alpha_{n} \rightarrow 0$ as $n \rightarrow \infty$, we have that

$$
v_{n}-x_{n} \rightarrow 0, v_{n}-y_{n} \rightarrow 0, x_{n}-z_{n} \rightarrow 0, z_{n}-u_{n} \rightarrow 0 \text { as } n \rightarrow \infty .
$$

Moreover, from (3.1), we also have

$$
\begin{aligned}
\left\|x_{n+1}-x_{n}\right\|^{2} & =\left\|\alpha_{n} u+\left(1-\alpha_{n}\right)\left[\beta_{n} y_{n}+\left(1-\beta_{n}\right) u_{n}\right]-x_{n}\right\|^{2} \\
& \leq \alpha_{n}\left\|x_{n}-u\right\|^{2}+\left(1-\alpha_{n}\right)\left[\beta_{n}\left\|y_{n}-x_{n}\right\|^{2}+\left(1-\beta_{n}\right)\left\|u_{n}-x_{n}\right\|^{2}\right] .
\end{aligned}
$$

This together with (3.8) implies that

$$
\left\|x_{n+1}-x_{n}\right\| \rightarrow 0 \text { as } n \rightarrow \infty \text {. }
$$

Furthermore, since $\left\{x_{n}\right\}$ is bounded in $H$, we can choose a subsequence $\left\{x_{n_{j}}\right\}$ of $\left\{x_{n}\right\}$ such that $x_{n_{j}} \rightarrow z$ and

$$
\limsup _{n \rightarrow \infty}\left\langle x_{n}-x^{*}, u-x^{*}\right\rangle=\lim _{j \rightarrow \infty}\left\langle x_{n_{j}}-x^{*}, u-x^{*}\right\rangle .
$$

This implies from (3.8) that $y_{n_{j}} \rightarrow z, u_{n_{j}} \rightarrow z, v_{n_{j}} \rightarrow z$ and $z_{n_{j}} \rightarrow z$ as $j \rightarrow \infty$.

Now, we show that $z \in F(T)$. From the definition of $v_{n_{j}}$ we have

$$
\left\langle y-v_{n_{j}}, T v_{n_{j}}\right\rangle-\frac{1}{r_{n_{j}}}\left\langle y-v_{n_{j}},\left(r_{n_{j}}+1\right) v_{n_{j}}-x_{n_{j}}\right\rangle \leq 0 \quad \forall y \in C .
$$


Put $z_{t}=t v+(1-t) z$ for all $t \in(0,1]$ and $v \in C$. Consequently, we get $z_{t} \in C$. From (3.10) and pseudocontractivity of $T$, it follows that

$$
\begin{aligned}
\left\langle v_{n_{j}}-z_{t}, T z_{t}\right\rangle \geq & \left\langle v_{n_{j}}-z_{t}, T z_{t}\right\rangle+\left\langle z_{t}-v_{n_{j}}, T v_{n_{j}}\right\rangle-\frac{1}{r_{n_{j}}}\left\langle z_{t}-v_{n_{j}},\left(1+r_{n_{j}}\right) v_{n_{j}}-x_{n_{j}}\right\rangle \\
= & -\left\langle z_{t}-v_{n_{j}}, T z_{t}-T v_{n_{j}}\right\rangle-\frac{1}{r_{n_{j}}}\left\langle z_{t}-v_{n_{j}}, v_{n_{j}}-x_{n_{j}}\right\rangle \\
& -\left\langle z_{t}-v_{n_{j}}, v_{n_{j}}\right\rangle \\
\geq & -|| z_{t}-\left.v_{n_{j}}\right|^{2}-\frac{1}{r_{n_{j}}}\left\langle z_{t}-v_{n_{j}}, v_{n_{j}}-x_{n_{j}}\right\rangle-\left\langle z_{t}-v_{n_{j}}, v_{n_{j}}\right\rangle \\
= & \left\langle v_{n_{j}}-z_{t}, z_{t}\right\rangle-\left\langle z_{t}-v_{n_{j}}, \frac{v_{n_{j}}-x_{n_{i}}}{r_{n_{j}}}\right\rangle .
\end{aligned}
$$

Since $v_{n}-x_{n} \rightarrow 0$ as $n \rightarrow \infty$, we obtain that $\frac{v_{n_{j}}-x_{n_{j}}}{r_{n_{j}}} \rightarrow 0$ as $j \rightarrow \infty$. Thus, as $j \rightarrow \infty$, it follows that $\left\langle z-z_{t}, T z_{t}\right\rangle \geq\left\langle z-z_{t}, z_{t}\right\rangle$. Hence $-\left\langle v-z, T z_{t}\right\rangle \geq-\left\langle v-z, z_{t}\right\rangle, \forall v \in C$. Letting $t \rightarrow 0$ and using the fact that $T$ is continuous, we obtain that $-\langle v-z, T z\rangle \geq-\langle v-z, z\rangle \forall v \in C$, which implies that $z=T z$.

Next, we show that $z \in E P(f)$. From (A2), we note that

$$
\frac{1}{r_{n}}\left\langle v-y_{n}, y_{n}-x_{n}\right\rangle \geq-f\left(y_{n}, v\right)=f\left(v, y_{n}\right) \forall v \in C,
$$

which implies that $f(v, z) \leq 0, \forall v \in C$. Put $z_{t}=t v+(1-t) z$ for all $t \in(0,1]$ and $v \in C$. Consequently, we get that $z_{t} \in C$ and $f\left(z_{t}, z\right) \leq 0$. Therefore, from (A1), we obtain that

$$
0=f\left(z_{t}, z_{t}\right) \leq t f\left(z_{t}, v\right)+(1-t) f\left(z_{t}, z\right) \leq t f\left(z_{t}, v\right) .
$$

Thus, $f\left(z_{t}, v\right) \geq 0, \forall v \in C$. Furthermore, as $t \rightarrow 0$, we have from (A3) that $f(z, v) \geq 0$, for all $v \in C$. This implies that $z \in E P(f)$.

Next, we show that $z \in V I(C, A)$. Since $A$ is Lipschitz continuous, we have

$$
\left\|A u_{n_{j}}-A z_{n_{j}}\right\| \rightarrow 0 \text { as } j \rightarrow \infty .
$$

Let

$$
B x= \begin{cases}A x+N_{C} x, & x \in C \\ \emptyset, & x \notin C,\end{cases}
$$

where $N_{C}(x)$ is the normal cone to $C$ at $x \in C$ given by

$$
N_{C}(x)=\{w \in H:\langle x-u, w\rangle \geq 0 \text { for all } u \in C\} .
$$

Then, $B$ is maximal monotone and $0 \in B x$ if and only if $x \in V I(C, A)$ (see, [14]). Let $(v, w) \in G(B)$. Then, we have $w \in B v=A v+N_{C} v$ and hence $w-A v \in N_{C} v$. Thus, we get $\langle v-u, w-A v\rangle \geq 0$, for all $u \in C$. On the other hand, since $u_{n_{j}}=P_{C}\left(x_{n_{j}}-\gamma_{n_{j}} A z_{n_{j}}\right)$ and $v \in C$, we have $\left\langle x_{n_{j}}-\gamma_{n_{j}} A z_{n_{j}}-u_{n_{j}}, u_{n_{j}}-v\right\rangle \geq 0$. Hence, $\left\langle v-u_{n_{j}},\left(u_{n_{j}}-x_{n_{j}}\right) / \gamma_{n_{j}}+A z_{n_{j}}\right\rangle \geq 0$. Thus, as $u_{n_{j}} \in C$, the above inequality implies that

$$
\begin{aligned}
\left\langle v-u_{n_{j}}, w\right\rangle & \geq\left\langle v-u_{n_{j}}, A v\right\rangle \\
& \geq\left\langle v-u_{n_{j}}, A v\right\rangle-\left\langle v-u_{n_{j}},\left(u_{n_{j}}-x_{n_{j}}\right) / \gamma_{n_{j}}+A z_{n_{j}}\right\rangle \\
& =\left\langle v-u_{n_{j}}, A v-A u_{n_{j}}\right\rangle+\left\langle v-u_{n_{j}}, A u_{n_{j}}-A z_{n_{j}}\right\rangle-\left\langle v-u_{n_{j}},\left(u_{n_{j}}-x_{n_{j}}\right) / \gamma_{n_{j}}\right\rangle \\
& \geq\left\langle v-u_{n_{j}}, A u_{n_{j}}-A z_{n_{j}}\right\rangle-\left\langle v-u_{n_{j}},\left(u_{n_{j}}-x_{n_{j}}\right) / \gamma_{n_{j}}\right\rangle .
\end{aligned}
$$


Therefore, we obtain that $\langle v-z, w\rangle \geq 0$. Then, the maximality of $B$ gives that $z \in B^{-1}(0)$. Therefore, $z \in V I(C, A)$. Using Lemma 2.4, we immediately obtain that

$$
\begin{aligned}
\limsup _{n \rightarrow \infty}\left\langle x_{n}-x^{*}, u-x^{*}\right\rangle & =\lim _{j \rightarrow \infty}\left\langle x_{n_{j}}-x^{*}, u-x^{*}\right\rangle \\
& =\left\langle z-x^{*}, u-x^{*}\right\rangle \leq 0 .
\end{aligned}
$$

It follows from (3.7), (3.13) and Lemma 2.5 that $x_{n} \rightarrow x^{*}=\Pi_{\mathscr{F}} u$.

Case 2. Suppose that there exists a subsequence $\left\{n_{i}\right\}$ of $\{n\}$ such that

$$
\left\|x_{n_{i}}-x^{*}\right\|^{2}<\left\|x_{n_{i}+1}-x^{*}\right\|^{2}
$$

for all $i \in \mathbb{N}$. Then, by Lemma 2.6, there exists a nondecreasing sequence $\left\{m_{k}\right\} \subset \mathbb{N}$ such that $m_{k} \rightarrow \infty$, and

$$
\left\|x_{m_{k}}-x^{*}\right\| \leq\left\|x_{m_{k}+1}-x^{*}\right\| \text { and }\left\|x_{k}-x^{*}\right\| \leq\left\|x_{m_{k}+1}-x^{*}\right\|,
$$

for all $k \in \mathbb{N}$. Now, from (3.7) and the facts that $\gamma_{n}<\frac{1}{L}$ for all $n \geq 0$ and $\alpha_{n} \rightarrow 0$ as $n \rightarrow \infty$, we get that $v_{m_{k}}-x_{m_{k}} \rightarrow 0, v_{m_{k}}-y_{m_{k}} \rightarrow 0, x_{m_{k}}-z_{m_{k}} \rightarrow 0$, and $z_{m_{k}}-u_{m_{k}} \rightarrow 0$ as $k \rightarrow \infty$. Thus, following the method in Case 1, we obtain

$$
\limsup _{k \rightarrow \infty}\left\langle x_{m_{k}}-x^{*}, u-x^{*}\right\rangle \leq 0
$$

Now, from (3.7), we have that

$$
\begin{aligned}
\left\|x_{m_{k}+1}-x^{*}\right\|^{2} \leq & \left(1-\alpha_{m_{k}}\right)|| x_{m_{k}}-x^{*} \|^{2}+2 \alpha_{m_{k}}\left\langle x_{m_{k}}-x^{*}, u-x^{*}\right\rangle \\
& +2 \alpha_{m_{k}}|| x_{m_{k}+1}-x_{m_{k}}\left|\left\|\mid u-x^{*}\right\| .\right.
\end{aligned}
$$

Hence, (3.14) and (3.16) imply that

$$
\begin{aligned}
\alpha_{m_{k}} \mid\left\|x_{m_{k}}-x^{*}\right\|^{2} \leq & \left\|x_{m k}-x^{*}\right\|^{2}-\left\|x_{m_{k}+1}-x^{*}\right\|^{2}+2 \alpha_{m_{k}}\left\langle x_{m_{k}}-x^{*}, u-x^{*}\right\rangle \\
& +2 \alpha_{m_{k}}|| x_{m_{k}+1}-x_{m_{k}}\left|\left\|\mid u-x^{*}\right\|\right. \\
\leq & 2 \alpha_{m_{k}}\left\langle x_{m_{k}}-x^{*}, u-x^{*}\right\rangle+2 \alpha_{m_{k}}|| x_{m_{k}+1}-x_{m_{k}}\left|\left\|\mid u-x^{*}\right\|,\right.
\end{aligned}
$$

which implies that

$$
\left\|x_{m_{k}}-x^{*}\right\|^{2} \leq 2\left\langle x_{m_{k}}-x^{*}, u-x^{*}\right\rangle+2|| x_{m_{k}+1}-x_{m_{k}}\left|\left\||| u-x^{*}\right\| .\right.
$$

Thus, using (3.9) and (3.15) we get that $\left\|x_{m_{k}}-x^{*}\right\| \rightarrow 0$ as $k \rightarrow \infty$. This together with (3.16) implies that $\left\|x_{m_{k}+1}-x^{*}\right\| \rightarrow 0$ as $k \rightarrow \infty$. But $\left\|x_{k}-x^{*}\right\| \leq\left\|x_{m_{k}+1}-x^{*}\right\|$ for all $k \in \mathbb{N}$ gives that $x_{k} \rightarrow x^{*}$. Therefore, from the above two cases, we can conclude that $\left\{x_{n}\right\}$ converges strongly to a point $x^{*}=\Pi_{\mathscr{F}} u$. The proof is complete.

If, in Theorem 3.1, we assume that $A=0$, then we obtain the following corollary.

Corollary 3.1. Let $C$ be a nonempty, closed and convex subset of a real Hilbert space $H$. Let $T: C \rightarrow H$ be a continuous pseudo-contractive mapping. Let $f: C \times C \rightarrow \mathbb{R}$ be a bifunction satisfying (A1)-(A4). Assume that $\mathscr{F}=F(T) \cap E P(f)$ is nonempty. Let $\left\{x_{n}\right\}$ be a sequence generated from an arbitrary $x_{0}, u \in C$ by

$$
x_{n+1}=\alpha_{n} u+\left(1-\alpha_{n}\right)\left[\beta_{n} y_{n}+\left(1-\beta_{n}\right) x_{n}\right] \text {, }
$$


where $y_{n}=F_{r_{n}} T_{r_{n}} x_{n}$ and $\left\{\beta_{n}\right\} \subset[e, 1) \subset(0,1)$. Then, $\left\{x_{n}\right\}$ converges strongly to a point $x^{*}$ in $\mathscr{F}$ which is nearest to $u$.

If, in Theorem 3.1, we assume that $T=I$, the identity mapping on $C$, then we obtain the following corollary.

Corollary 3.2. Let $C$ be a nonempty, closed and convex subset of a real Hilbert space H. Let $A: C \rightarrow H$ be a L-Lipschitz monotone mapping with Lipschitz constant L. Let $f: C \times C \rightarrow \mathbb{R}$ be a disfunction satisfying (A1)-(A4). Assume that $\mathscr{F}=V I(C, A) \cap E P(f)$ is nonempty. Let $\left\{x_{n}\right\}$ be a sequence generated from an arbitrary $x_{0}, u \in C$ by

$$
\left\{\begin{array}{l}
z_{n}=P_{C}\left[x_{n}-\gamma_{n} A x_{n}\right] \\
x_{n+1}=\alpha_{n} u+\left(1-\alpha_{n}\right)\left[\beta_{n} y_{n}+\left(1-\beta_{n}\right) u_{n}\right],
\end{array}\right.
$$

where $y_{n}=F_{r_{n}} x_{n}, u_{n}=P_{C}\left[x_{n}-\gamma_{n} A z_{n}\right],\left\{\gamma_{n}\right\} \subset[a, b] \subset\left(0, \frac{1}{L}\right)$ and $\left\{\beta_{n}\right\} \subset[e, 1) \subset(0,1)$. Then, $\left\{x_{n}\right\}$ converges strongly to a point $x^{*}$ in $\mathscr{F}$ which is nearest to $u$.

If, in Theorem 3.1, we assume that $f=0$, then we obtain the following corollary.

Corollary 3.3. Let $C$ be a nonempty, closed and convex subset of a real Hilbert space H. Let $T: C \rightarrow H$ be a continuous pseudocontractive mapping and $A: C \rightarrow H$ be a L-Lipschitz monotone mapping with Lipschitz constant $L$. Assume that $\mathscr{F}=F(T) \cap V I(C, A)$ is nonempty. Let $\left\{x_{n}\right\}$ be a sequence generated from an arbitrary $x_{0}, u \in C$ by

$$
\left\{\begin{array}{l}
z_{n}=P_{C}\left[x_{n}-\gamma_{n} A x_{n}\right], \\
x_{n+1}=\alpha_{n} u+\left(1-\alpha_{n}\right)\left[\beta_{n} y_{n}+\left(1-\beta_{n}\right) u_{n}\right],
\end{array}\right.
$$

where $y_{n}=T_{r_{n}} x_{n}, u_{n}=P_{C}\left[x_{n}-\gamma_{n} A z_{n}\right],\left\{\gamma_{n}\right\} \subset[a, b] \subset\left(0, \frac{1}{L}\right)$ and $\left\{\beta_{n}\right\} \subset[e, 1) \subset(0,1)$. Then, $\left\{x_{n}\right\}$ converges strongly to a point $x^{*}$ in $\mathscr{F}$ which is nearest to $u$.

If, in Theorem 3.1, we assume that $f=A=0$, then we obtain the following corollary.

Corollary 3.4. Let $C$ be a nonempty, closed and convex subset of a real Hilbert space H. Let $T: C \rightarrow H$ be a continuous pseudocontractive mapping. Assume that $F(T)$ is nonempty. Let $\left\{x_{n}\right\}$ be a sequence generated from an arbitrary $x_{0}, u \in C$ by

$$
x_{n+1}=\alpha_{n} u+\left(1-\alpha_{n}\right)\left[\beta_{n} T_{r_{n}} x_{n}+\left(1-\beta_{n}\right) x_{n}\right],
$$

where $\left\{\beta_{n}\right\} \subset[e, 1) \subset(0,1)$. Then, $\left\{x_{n}\right\}$ converges strongly to a point $x^{*}$ in $F(T)$ which is nearest to $u$.

From Theorem 3.1, we can also obtain the following result on the common minimum norm solution for the fixed point problem of a continuous pseudocontractive mapping, the variational inequality problem for Lipschitz monotone mappings and the equilibrium problem.

Theorem 3.2. Let $C$ be a nonempty, closed and convex subset of a real Hilbert space $H$. Let $T: C \rightarrow H$ be a continuous pseudocontractive mapping and $A: C \rightarrow H$ be a L-Lipschitz monotone mapping with Lipschitz constant L. Let $f: C \times C \rightarrow \mathbb{R}$ be a bifunction satisfying (Al)-(A4). Assume that $\mathscr{F}=F(T) \cap$ $V I(C, A) \cap E P(f)$ is nonempty. Let $\left\{x_{n}\right\}$ be a sequence generated from an arbitrary $x_{0} \in C$ by

$$
\left\{\begin{array}{l}
z_{n}=P_{C}\left[x_{n}-\gamma_{n} A x_{n}\right] \\
x_{n+1}=P_{C}\left[\left(1-\alpha_{n}\right)\left(\beta_{n} y_{n}+\left(1-\beta_{n}\right) u_{n}\right)\right]
\end{array}\right.
$$


where $y_{n}=F_{r_{n}} T_{r_{n}} x_{n}, u_{n}=P_{C}\left[x_{n}-\gamma_{n} A z_{n}\right],\left\{\gamma_{n}\right\} \subset[a, b] \subset\left(0, \frac{1}{L}\right)$ and $\left\{\beta_{n}\right\} \subset[e, 1) \subset(0,1)$. Then, $\left\{x_{n}\right\}$ converges strongly to a minimum norm point $x^{*}$ of $\mathscr{F}$.

Remark 3.1. Theorem 3.1 extends Theorem 3.1 and 4.1 of Tada and Takahashi [15] and Theorem 3 of Kumam [8] to a more general class of continuous pseudocontractive and monotone mappings. Our scheme does not involve computation of $C_{n}$ and $Q_{n}$ to obtain $x_{n+1}$ for each $n \geq 1$. Corollary 3.3 extends Theorem 3.1 of Nadezhkina and Takahashi [12] and Theorem 3.1 of Zegeye and Shahzad [25] to a general class of continuous pseudocontractive mapping and Lipschitz monotone mappings. Our results provide affirmative answers to the questions raised in Section 1.

\section{THE NUMERICAL EXAMPLE}

In this section, we give an example of a continuous pseudocontractive mapping $T$, a Lipschitz monotone mapping $A$ and a bifunction $f$ satisfying (A1)-(A4) with all the conditions of Theorem 3.1 and a numerical experiment result to support the conclusion of the theorem.

Example 4.1. Let $H=\mathbb{R}$ with the Euclidean norm. Let $C=[-1,10]$ and let $T: C \rightarrow \mathbb{R}$ be a mapping defined by

$$
T x:= \begin{cases}-4 x-\frac{3}{2}, & x \in\left[-1,-\frac{1}{2}\right), \\ x, & x \in\left[-\frac{1}{2}, 10\right] .\end{cases}
$$

Then, we see that $(I-T)$ is continuous and monotone and hence $T$ is a continuous pseudocontractive mapping on $C$. In addition, if $x \in\left[-1,-\frac{1}{2}\right)$, and $z \in\left[-1, \frac{1}{2}\right)$, we have that

$$
\langle y-z, T z\rangle-\frac{1}{r}\langle y-z,(1+r) z-x\rangle \leq 0, \quad \forall y \in C,
$$

is equivalent to

$$
\left[(1+r) z-x+\left(4 z r+\frac{3}{2} r\right)\right] y \geq\left[(1+r) z-x+\left(4 r z+\frac{3}{2} r\right)\right] z, \quad \forall y \in C .
$$

But this holds, if $z=\frac{x-\frac{3 r}{2}}{1+5 r}$. If $x \in\left[-\frac{1}{2}, 10\right]$, we get from $z \in\left[-\frac{1}{2}, 10\right]$ that

$$
\langle y-z, T z\rangle-\frac{1}{r}\langle y-z,(1+r) z-x\rangle \leq 0, \quad \forall y \in C,
$$

is equivalent to $(y-z) z-\frac{1}{r}(y-z)[(1+r) z-x] \leq 0, \forall y \in C$, which is further equivalent to $(z-x) y \geq$ $(z-x) z, \forall y \in C$. But this holds, if $z=x$. Therefore, we get that

$$
T_{r_{n}} x:= \begin{cases}\frac{x-\frac{3 r}{2}}{1+5 r}, & x \in\left[-1,-\frac{1}{2}\right), \\ x, & x \in\left[-\frac{1}{2}, 10\right] .\end{cases}
$$

Let $A: C \rightarrow \mathbb{R}$ be a mapping defined by

$$
A x:= \begin{cases}0, & x \in[-1,1], \\ (x-1)^{2}, & x \in(1,10] .\end{cases}
$$

Then, we easily see that $A$ is monotone. Now, we show that $A$ is Lipschitz.

Case 1: If $x, y \in[-1,1]$, then

$$
|A x-A y|=|0-0| \leq|x-y| .
$$


Case 2: If $x \in[-1,1]$ and $y \in(1,10]$, then

$$
\begin{aligned}
|A x-A y| & =\left|0-(y-1)^{2}\right|=(y-1)^{2} \leq|y-x|^{2}=|x-y|^{2} \\
& \leq|x+y||x-y| \leq 11|x-y| .
\end{aligned}
$$

Case 3: If $x, y \in(1,10]$, then

$$
\begin{aligned}
|A x-A y| & =\left|(x-1)^{2}-(y-1)^{2}\right| \\
& \leq\left|x^{2}-y^{2}\right|+2|x-y| \\
& \leq|x+y||x-y|+2|x-y| \\
& \leq 20|x-y|+2|x-y|=22|x-y| .
\end{aligned}
$$

From Cases 1, 2 and 3, we obtain that $A$ is Lipschitz with Lipschitz constant $L=22$.

Let $f: C \times C \rightarrow \mathbb{R}$ be defined by

$$
f(x, y):= \begin{cases}0, & x \in[-1,0), \\ 2 x y-2 x^{2}, & x \in(0,10] .\end{cases}
$$

Then, we observe that $f(x, x)=0, f(x, y)+f(y, x) \leq 0, \lim _{t \rightarrow 0} f(t z+(1-t) x, y) \leq f(x, y)$ for all $x, y, z \in C$ and for each $x \in C, y \rightarrow f(x, y)$ is convex and lower semicontinuous. Furthermore, if $x \in[-1,0)$, the inequality

$$
F_{r} x=\left\{z \in C: f(z, y)+\frac{1}{r}\langle y-z, z-x\rangle \geq 0, \quad \forall y \in C\right\},
$$

shows that we may take $F_{r}(x)=x$ and if $x \in[0,10]$, we obtain from (4.1) that

$$
2 r\left(z y-z^{2}\right)+(y-z)(z-x) \geq 0, \quad \forall y \in C,
$$

which implies that $F_{r}(x)=z=\frac{x}{2 r+1}$. Hence,

$$
F_{r}(x):= \begin{cases}x, & x \in[-1,0) \\ \frac{x}{2 r+1}, & x \in[0,10]\end{cases}
$$

It is also clear that $F(T) \cap V I(C, A) \cap E F(f)=\left[-\frac{1}{2}, 10\right] \cap[-1,1] \cap[-1,0]=\left[-\frac{1}{2}, 0\right]$. If $\alpha_{n}=\frac{1}{n+100}$, $\gamma_{n}=\frac{1}{n+100}+0.01, \beta_{n}=\frac{1}{2 n+100}+0.05, r_{n}=10, \forall n \geq 1$, and $u=4.0$, then the conditions of Theorem 3.1 are satisfied and iterative scheme (3.1) is reduced to

$$
\left\{\begin{array}{l}
z_{n}=P_{C}\left[x_{n}-\gamma_{n} A x_{n}\right] \\
x_{n+1}=\alpha_{n} u+\left(1-\alpha_{n}\right)\left[\beta_{n} y_{n}+\left(1-\beta_{n}\right) u_{n}\right]
\end{array}\right.
$$

where $y_{n}=F_{r_{n}} T_{r_{n}} x_{n}$ and $u_{n}=P_{C}\left[x_{n}-\gamma_{n} A z_{n}\right]$. Thus, for $x_{0}=-1.0$, the sequence generated in iterative scheme (4.2) converges strongly to $0=P_{\mathscr{F}}(u)$. See the following table and Figure

\begin{tabular}{|c|c|c|c|c|c|c|c|c|}
\hline $\mathrm{n}$ & 0 & 100 & 200 & 500 & 1000 & 2000 & 3000 & 4000 \\
\hline$x_{n}$ & -1.0000 & 0.00426 & 0.0211 & 0.0084 & 0.0042 & 0.0021 & 0.0014 & 0.00001 \\
\hline
\end{tabular}




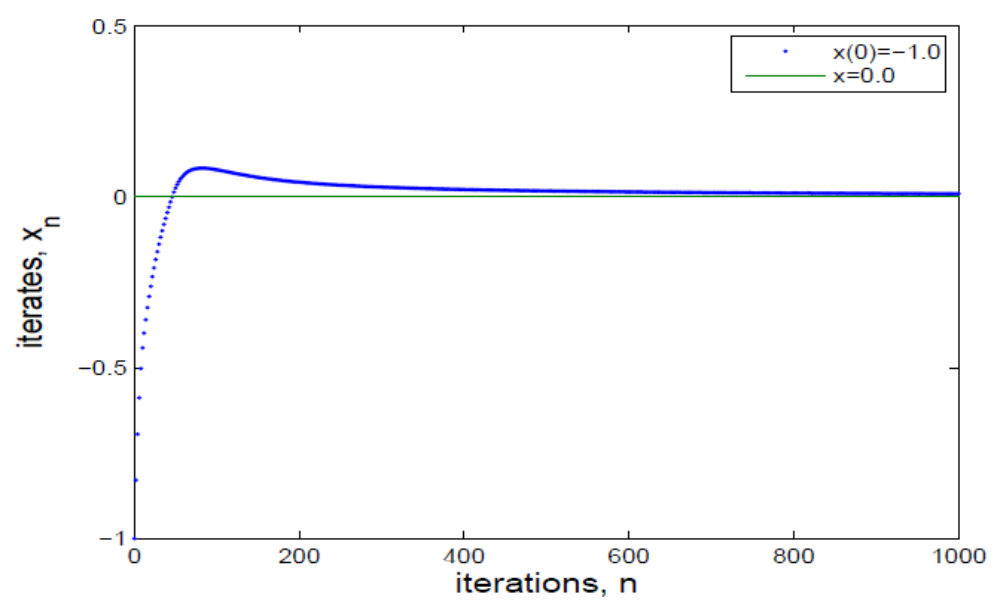

\section{REFERENCES}

[1] E. Blum, W. Oettli, From optimization and variational inequalities to equilibrium problems, Math. Student 63 (1994), 123-145.

[2] G. Cai, S. Bu, An iterative algorithm for a general system of variational inequalities and fixed point problems in quniformly smooth Banach spaces, Optim. Lett. 7 (2013), 267-287.

[3] H. Iiduka, W. Takahashi, M. Toyoda, Approximation of solutions of variational inequalities for monotone mappings, PanAmer Math. J. 14 (2004), 49-61.

[4] H. Iiduka, W. Takahashi, Strong convergence theorems for nonexpansive mappings and inverse-strongly monotone mappings, Nonlinear Anal. 61 (2005), 341-350.

[5] H. Iiduka, W. Takahashi, Strong convergence studied by a hybrid type method for monotone operators in a Banach space, Nonlinear Anal. 68 (2008), 3679-3688.

[6] S. Kamimura, W. Takahashi, Strong convergence of proximal-type algorithm in a Banach space, SIAM J. Optim. 13 (2002), 938-945.

[7] D. Kinderlehrer, G. Stampaccia, An iteration to variational inequalities and their applications, Academic Press, New York, 1990.

[8] P. Kumam, A hybrid approximation method for equilibrium and fixed point problems for a monotone mapping and a nonexpansive, Nonlinear Anal.: Hybrid Syst. 2 (2008), 1245-1255.

[9] J.L. Lions, G. Stampacchia, Variational inequalities, Commun. Pure Appl. Math. 20 (1967), 493-517.

[10] A. Moudafi, Weak convergence theorems for nonexpansive mappings and equilibrium problems, J. Nonlinear Convex Anal. 9 (2008), 37-43.

[11] P. E. Maingé, Strong convergence of projected subgradient methods for nonsmooth and non-strictly convex minimization, Set-Valued Anal. 16 (2008), 899-912.

[12] N. Nadezhkina, W. Takahashi, Weak convergence theorem by an extragradient method for nonexpansive mappings and monotone mappings, J. Optim. Theory Appl. 128 (2006), 191-201.

[13] X. Qin, Y. J. Cho, S. M. Kang, convergence theorems of common elements for equilibrium problems and fixed point problem in Banach spaces, J. Comput. Appl. Math. 225 (2009), 20-30.

[14] R. T. Rockafellar, On the maximality of sums of nonlinear monotone operators, Trans. Amer. Math. Soc. 149 (1970), 75-88.

[15] A. Tada, W. Takahashi, Weak and strong convergence theorems for nonexpansive mappings and equilbrium problems, J. Optim. Theory Appl. 133 (2007), 359-370.

[16] W. Takahashi, K. Zembayashi, Strong and weak convergence theorems for equilibrium problems and relatively nonexpansive mappings in Banach spaces, Nonlinear Anal. 70 (2009), 45-57.

[17] W. Takahashi, Nonlinear Functional Analysis, Yokohama Publishers, Yokohama, 2000.

[18] H.K. Xu, Another control condition in an iterative method for nonexpansive mappings, Bull. Aust. Math. Soc. 65 (2002), 109-113. 
[19] H. Zegeye, N. Shahzad, Y. Yao, Minimum-norm solution of variational inequality and fixed point problem in Banach spaces, Optimization, 64 (2015), 453-471.

[20] H. Zegeye, E.U. Ofoedu, N. Shahzad, Convergence theorems for equilibrium problem, variotional inequality problem and countably infinite relatively quasi-nonexpansive mappings, Appl. Math. Comput. 216 (2010), 3439-3449.

[21] H. Zegeye, N. Shahzad, Strong convergence for monotone mappings and relatively weak nonexpansive mappings, Nonlinear Anal. 70 (2009), 2707-2716.

[22] H. Zegeye, An iterative approximation method for a common fixed point of two pseudocontractive mappings, ISRN Math. Anal. 2011 (2011), Article ID 621901.

[23] H. Zegeye, N. Shahzad, Convergence of Mann's type iteration method for generalized asymptotically nonexpansive mappings, Comput. Math. Appl. 62 (2011), 4007-4014.

[24] H. Zegeye, N. Shahzad, A hybrid scheme for finite families of equilibrium, variational inequality and fixed point problems, Nonlinear Anal. 74 (2011), 263-272.

[25] H. Zegeye, N. Shahzad, Solutions of variational inequality problems in the set of fixed points of pseudocontractive mappings, Carpathian J. Math. 30 (2014), 257-265. 